\title{
Editorial
}

\section{A psicopatologia sob a perspectiva do sujeito singular}

Mario Eduardo Costa Pereira*1

O termo "psicopatologia" jamais fez parte da língua comum. Trata-se de um neologismo cuja criação é atribuída ao médico alemão Hermann Emminghaus (1845-1904) que o teria utilizado pela primeira vez em 1878 como sinônimo de "psiquiatria clínica". Sua imersão no campo psiquiátrico é, por assim dizer, sua marca de nascença.

Uma segunda característica dessa palavra é sua aparente autoevidência. De hábito, tende a evocar espontaneamente a ideia do estudo das "doenças mentais", a despeito do caráter profundamente problemático dessa expressão, tantas vezes já assinalado: se se trata de uma "doença" (fenômeno da res extensa cartesiana) como pode, então, ser "mental" (res cogitans)? E se é "mental", como pode ser "doença"? E que ciência é essa que toma por objeto uma das entidades mais metafisicas que se possa conceber: a alma (psique)? Mesmo assim — e uma vez mais — ao remeter

\footnotetext{
${ }^{* 1}$ Universidade Estadual de Campinas - Unicamp (Campinas, SP, Brasil) .
} 
de forma intuitiva ao âmbito semântico da "doença", ainda que problematicamente qualificada de "mental", reencontramos aqui a pregnância da matriz médico-psiquiátrica associada à psicopatologia. Ou seja, essa disciplina racional (trata-se de uma modalidade de "logos") teria como referência a "doença mental", sendo esta formulada de modo a constituir um objeto positivamente acessível aos métodos das ciências empírico-experimentais e, eventualmente, abordável sob a perspectiva biológica própria ao campo médico.

Vertentes mais humanistas, ou mesmo psicanalíticas, tendem a "despsiquiatrizar" a "psicopatologia", referindo-a não mais à objetividade da natureza (da Physis), mas agora ao âmbito fortemente subjetivo do "sofrimento psíquico". Sob essa ótica, a "subjetividade" e a própria noção de "sujeito" são convocadas ao centro da elucidação racional do pathos humano. É assim que, já em 1926, Viktor von Weizsäcker, pioneiro da chamada Antropologia Médica, alertava para o fato de que, para além das fronteiras psiquiátricas, era o conjunto do campo médico que permanecia distante de uma interrogação sistemática sobre o sujeito do qual se ocupa: "É surpreendente, mas na verdade, é um fato que não se pode negar, que a medicina moderna não tem nenhuma doutrina sobre o homem doente" (Weizsäcker, 1926/1987, p. 72). É imperativo, portanto, sustentava Weizsäcker, incorporar a subjetividade do paciente à realidade objetiva a ser tratada. Vê-se assim que o estatuto do pathos - tema central da psicopatologia — interroga não apenas o campo psiquiátrico e da chamada "saúde mental", mas o conjunto da Medicina e das práticas clínicas.

É importante destacar aqui que essas perspectivas que se propõem a estudar o pathos sob a ótica da subjetividade não implicam, necessariamente, a adoção de uma teoria "psicogênica" da "doença", fosse ela qualificada ou não de "mental". Trata-se, antes, de se examinar o fenômeno do adoecimento sob o prisma da subjetividade. Dessa forma, é necessário destacar claramente a "patologia", fenômeno que pressupõe um sujeito, do campo da "nosologia", ou seja, a doença tomada enquanto perturbação biológica prejudicial ao funcionamento fisiológico ótimo, típico de uma espécie natural (Boorse, 1977). Vemos aqui se delinearem mais claramente três grupos de problemas decisivos para o âmbito da "patologia": 1) a "nosologia", enquanto estudo das disfunções naturais capazes de incidir de forma mórbida na realização ótima de funções biológicas necessárias à sobrevivência do indivíduo e da espécie; 2) a "patologia" propriamente dita em termos semânticos: o estudo das perturbações ou impedimentos da realização possível de um sujeito singular no interior do laço social (Pereira, 2019) e 3) o estudo das possíveis incidências e interações 


\section{EDITORIAL}

de um campo sobre o outro: a nosologia sobre a patologia, a patologia sobre a nosologia e as margens de autonomia de uma em relação à outra.

Acompanhando esse desdobramento da premissa de von Weizsäcker, segundo a qual a patologia se refere necessariamente ao âmbito do sujeito, então o próprio termo de "psico"-patologia soaria excessivo e redundante: qual a razão para se sobrecarregar um fenômeno intrinsecamente subjetivo — já evocado pelo radical grego "pathos" - de uma referência à "alma" (psique)? Mesmo assim — ou por isso mesmo — o influente autor alemão não empregará a noção de "patologia" para designar o estudo do adoecimento sob a ótica do sujeito. Esta já se encontra fortemente impregnada da referência tradicional da doença ao campo da natureza, enquanto perturbação mórbida da fisiologia. Além disso, decorre de seu pensamento que o tipo de racionalidade necessária para o entendimento apropriado da subjetividade de "homem doente" não é o "logos" das ciências naturais, mas aquela própria à filosofia. Daí sua proposta de uma "patosofia" (Weizsäcker, 1956/2011), como disciplina racional visando conhecer o pathos do homem doente, considerado em sua condição de sujeito (cf. Pereira, 2014).

Esse estado de coisas constitui um paradoxo, talvez até mesmo uma contradição: a psicopatologia é a disciplina nuclear (Banzato \& Zorzanelli, 2020) não apenas do campo da psiquiatria, mas também fundamento de todas as práticas clínicas do campo da chamada "saúde mental". Dela depende a delimitação do elemento mórbido em jogo, do mal a ser tratado e, por extensão, da direção e dos objetivos do tratamento.

Caso especial nesse debate é constituído pela psicanálise. É muito significativo notar que mesmo Freud, com todo seu rigor teórico, jamais propôs uma definição formalmente conceitual para "psicopatologia", nem mesmo em sua "Psicopatologia da vida cotidiana" (1901/1987b). Tampouco no capítulo II de seu "Projeto para uma psicologia científica" (1950[1895]/1987a), intitulado precisamente como "Psicopatologia". O respeitadíssimo Vocabulário da Psicanálise não lhe consagra verbete; nenhum destaque lhe é dado na biografia de Freud redigida por Jones. Mesmo assim, a teoria freudiana é aquela que provavelmente mais avançou em relação à necessidade de se estudar a patologia enquanto fenômeno relativo a um sujeito singular: a técnica da livre-associação sob transferência permite evidenciar as redes associativas relativas à produção das significações próprias a cada sujeito, o que faz com que jamais um sonho possa ser interpretável através de um "dicionário geral dos sonhos"; as modalidades de satisfação erótica dependem da fantasia inconsciente de cada um; as ideias de "equação etiológica" e de "séries complementares" 
permitem conceber de forma individualizada a eclosão da patologia mental em um sujeito singular, sem que se necessite recusar a participação de fatores naturais nos processos nela implicados etc. Nesse sentido, a psicanálise, ainda que tradicionalmente não tenha formalizado explicitamente uma psicopatologia nos termos de von Weizsäcker, orienta-se nesse campo precisamente por noções como as de conflito, de defesa e de satisfação erótica como necessariamente relativas a um sujeito singular. E mais: um sujeito incarnado corporalmente. Como diria de maneira límpida Lacan, ao tratar justamente das relações entre psicanálise e medicina: o gozo é um fenômeno do corpo (Lacan, 1966).

O caráter específico da abordagem psicanalítica no que tange à subjetividade própria aos fenômenos psicopatológicos torna-se ainda mais evidente quando a comparamos a uma outra tradição teórica fundamental nesse campo: a fenomenologia jasperiana. Este esforço teórico para a constituição da psicopatologia como disciplina científica formalmente estruturada e autônoma em relação à psiquiatria surge no começo do século $\mathrm{XX}$, com a publicação da Allgemaine Psychopathologie (Psicopatologia Geral), de Karl Jaspers, em 1913. Nesse célebre tratado, a questão da subjetividade ocupa lugar central: trata-se de estudar os fenômenos psíquicos patológicos do ponto de vista das vivências subjetivas (Erlebnis) a eles tipicamente associadas: "O objeto da psicopatologia é o fenômeno psíquico realmente consciente. Queremos saber o que os homens vivenciam (Erlebnis) e como o fazem" (Jaspers, 1913/1979, p. 13). Dessa forma, o que se visa em primeiro lugar é a descrição das experiências típicas conscientemente vividas em um estado mental mórbido específico. Ou seja, apesar de visar a experiência subjetiva, o método da Psicopatologia Geral pretende alcançar um estatuto de objetividade: "conhecer a envergadura das realidades psíquicas" (p. 13). Em outros termos, a subjetividade visada deve, sob a ótica de Jaspers, corresponder às exigências científicas de se produzir, com razão e método, conhecimentos objetivos de validade universal: não se trata, portanto, de descrever a subjetividade singular de um sujeito acometido de uma condição patológica especifica, mas as características típicas e generalizáveis da experiência conscientemente vivida em cada tipo específico de condição psicopatológica. $\mathrm{O}$ foco não é, pois, a singularidade da condição patológica de cada sujeito, mas a descrição da tipicidade generalizável de cada entidade psicopatológica, seu nível propriamente transcendental (para empregar um termo caro à fenomenologia de Husserl, sobre a qual se inspira o psiquiatra-filósofo de Heidelberg).

Cabe destacar que o método jasperiano não visa fundamentalmente fornecer uma psicologia patológica, no sentido de Ribot, ou seja, explicitar como os processos mentais tidos como normais podem vir a se tornar 


\section{EDITORIAL}

mórbidos. Para Jaspers, o ponto de partida são as próprias condições doentias já tradicionalmente definidas, tomadas como referências para a aplicação do método fenomenológico. Afirma explicitamente a Allgemeine Psychopathologie: "Mas nem todos os fenômenos psíquicos constituem nosso objeto. Apenas os "patológicos"” (p. 13). Em síntese, partindo de fenômenos mentais tidos a priori e tradicionalmente como "patológicos", Jaspers propõe o emprego de um método fenomenológico capaz de descrever em um plano abstrato (na verdade, transcendental) as experiências vividas (Erlebnis) típicas e generalizáveis de cada entidade patológica. Daí a proposta jasperiana de uma Psicopatologia da subjetividade não tomada como singular, mas como Geral.

Ora, como vimos, a psicopatologia desempenha um papel epistemológico e técnico decisivo para a psiquiatria, para a psicanálise e para todas as demais práticas clínicas no campo da chamada saúde mental, estabelecendo aquilo que constitui o foco mórbido visado pelo tratamento. Contemporaneamente, diferentes princípios organizadores competem pelo prestígio de descrever as condições a serem consideradas patológicas e, consequentemente, pela determinação dos meios legítimos de tratamento e de seus objetivos terapêuticos. Encontramos, entre os mais relevantes atualmente, princípios naturalistas (RDoC), empiricistas (Evidence-Based Psychiatry); prático-convencionais (DSM), valorativos (Values-Basic Psychiatry); fenomenológicos (psicopatologia fenomenológica) existenciais (Daseinanalyse); subjetivo-singulares (psicanálise). A este último, acrescentaria a (Psico) Patologia (do sujeito) que vimos desenvolvendo desde 2013 no contexto do Laboratório de Psicopatologia - Sujeito e Singularidade (LaPSuSUNICAMP), que se propõe não a constituir uma nova "psicopatologia psicanalítica", mas se servir da concepção de sujeito decorrente da psicanálise de Freud e Lacan para fundamentar uma (psico)patologia sob a ótica das perturbações da subjetividade em sua dimensão propriamente singular. ${ }^{1}$ A psicopatologia fundamental proposta por Pierre Fédida (1998) preocupa-se, a um só tempo, com a delimitação do pathos psíquico implicado na psicopatologia,

${ }^{1}$ Trata-se, portanto, de uma nova definição de Psicopatologia, pertinente não apenas à psicanálise, mas a todo o campo das práticas clínicas, concebendo-a como o estudo dos impasses mórbidos perturbando, ou mesmo impedindo, a realização de um sujeito singular de forma responsável (levando em consideração a alteridade e a civilização) no interior do laço social (Pereira, 2019). 
como das modalidades de interação dos diferentes modelos científicos que se entrecruzam nessa encruzilhada epistemológica que caracteriza o campo do psicopatológico.

Cada um dos princípios organizadores acima mencionados dispõe de seus próprios operadores internos que lhes confere sustentação racional própria. Contudo, a questão da escolha daquele que deveria desempenhar o papel de organizador último da concepção de patologia e das práticas clínico-terapêuticas dela derivadas dependem fundamentalmente de critérios éticos. Diferentes abordagens técnicas produzem diferentes alterações no plano mundano e concreto relacionado à patologia, provocando diferentes incidências nos impasses patológicos subjacentes. A tomada de decisão quanto à referência psicopatológica a ser escolhida (com as consequentes finalidades terapêuticas dela decorrente) não depende de critérios técnicos e científicos, mas um posicionamento propriamente ético. A ciência e a técnica são colocadas a serviço da ética. Não o contrário. A política e as diferentes estratégias empregadas para alcançar os objetivos clínico-terapêuticos dependem igualmente da concepção ética embutida em cada (psico)patologia e em cada concepção de Terapeia a ela associada.

Dessa forma, os desafios epistemológicos, científicos e técnicos do campo da (psico)patologia permanecem totalmente vinculados a seus métodos próprios, mas dependentes e orientados por uma ética que os fundamente, justifique e oriente. Ao considerarmos - eticamente - a psicopatologia um fenômeno que incide sobre um sujeito tomado enquanto tal, perturbando ou impedindo suas possibilidades de realização no interior do laço social, então podemos encontrar na ética psicanalítica o fundamento e o organizador desse campo. Uma proposição de Lacan sobre a justificativa moral das intervenções no campo da clínica e das ações terapêuticas, talvez sintetize os fundamentos éticos de uma (psico)patologia orientada pela subjetividade singular: "É certo que nossa justificativa, assim como nosso dever, é melhorar a posição do sujeito...” (Lacan, 1962-63/2004, p. 70; a tradução é de minha autoria). Essa perspectiva ética permite definir o que constitui impasse patológico do ponto de vista do sujeito e estabelecer a direção e objetivos do tratamento, de modo que diferentes tipos de técnica podem ser agenciados e orquestrados tendo como orientação e justificativa a contribuição que possam eventualmente trazer no sentido dos objetivos éticos do tratamento. No caso, uma psicopatologia do sujeito singular conduzindo a uma clínica da melhora da posição do sujeito de maneira responsável com a civilização. 


\section{EDITORIAL}

\section{Referências}

Banzato, C. E. M., \& Zorzanelli, R. T. (2020, jun.). Making sense of the subjective experience of others. Revista Latinoamericana de Psicopatologia Fundamental, 23(2), 337-348. Available from: <http:// www.scielo.br/scielo.php?script=sci_arttext\&pid=S1415-47142020000200337\&ln $\mathrm{g}=\mathrm{en} \& \mathrm{nrm}=\mathrm{iso}>$.

Boorse, C. (1977). Health as a theoretical concept. Philosophy of science, 44, 542$-573$.

Fédida, P. (1998, set.). De uma psicopatologia fundamental - Nota sobre a noção de paradigma. Revista Latinoamericana de Psicopatologia Fundamental, I(3), 102-121.

Freud, S. (1987a). Projeto para uma psicologia científica. In Edição Standard Brasileira das Obras Psicológicas Completas de Sigmund Freud (Vol. I). Rio de Janeiro, RJ: Imago. (Trabalho original publicado em 1950[1895]).

Freud, S. (1987b). Psicopatologia da vida quotidiana. In Edição Standard Brasileira das Obras Psicológicas Completas de Sigmund Freud (Vol. VI). Rio de Janeiro, RJ: Imago. (Trabalho original publicado em 1901).

Jaspers, K. (1979). Psicopatologia Geral. Rio de Janeiro, RJ: Atheneu. (Trabalho original publicado em 1913).

Lacan, J. (1966). Conferência: O lugar da psicanálise na medicina. <http://pt.scribd. com/doc/167248091/Lacan-O-Lugar-da-Psicanalise-na-Medicina>.

Lacan, J. (2004). Le séminaire. Livre X. L'angoisse. Paris, FR: Éditions du Seuil. (Trabalho original publicado em 1962-63).

Pereira, M. E. C. (2014). A crise da psiquiatria centrada no diagnóstico e o futuro da clínica psiquiátrica: psicopatologia, antropologia e o sujeito da psicanálise. Physis: Revista de Saúde Coletiva, 24, 1035-1052. <https://www.scielo.br/j/ physis/a/pFvkCC4j98w5vmYvJJ4tCxm/?format=pdf\&lang=pt>.

Pereira, M. E. C. (2019, dez.). Projeto de uma (psico)patologia do sujeito (I): redefinição do conceito de psicopatologia à luz da questão do sujeito. Revista Latinoamericana de Psicopatologia Fundamental, 22(4), 828-858. <http://www. scielo.br/pdf/rlpf/v22n4/1415-4714-rlpf-22-04-0828.pdf>.

Von Weizsäcker, V. (1987). Der Arzt und der Kranke; Stücke einer medizinischen Anthropologie". In Gesammelte Schriften (Bd.5; pp. 69-86). Frankfurt Am Main: Suhrkamp. (Trabalho original publicado em 1926).

Von Weizsäcker, V. (2011). Pathosophie. Grenoble: Jérôme Millon, 2011. (Trabalho original publicado em 1956). 
Citação/Citation: Pereira, M. E. C. (2021, set.). Editorial. A psicopatologia sob a perspectiva do sujeito singular. Revista Latinoamericana de Psicopatologia Fundamental, 24(3), 501-508. http://dx.doi.org/10.1590/1415-4714.2021v24n3p501.1.

Editoras/Editors: Prof. Dr. Nelson da Silva Junior, Profa. Dra. Maria Livia Tourinho Moretto, Profa. Dra. Ana Maria Galdini R. Oda, Profa. Dra. Sonia Leite

Recebido/Received: 23.8.2021 / 8.23.2021

Aceito/Accepted: $25.8 .2021 / 8.25 .2021$

Copyright: (C) 2009 Associação Universitária de Pesquisa em Psicopatologia Fundamental/ University Association for Research in Fundamental Psychopathology. Este é um artigo de livre acesso, que permite uso irrestrito, distribuição e reprodução em qualquer meio, desde que o autor e a fonte sejam citados / This is an open-access article, which permits unrestricted use, distribution, and reproduction in any medium, provided the original authors and sources are credited.

\section{Mario Eduardo Costa Pereira}

Psiquiatra; Psicanalista; Professor titular de Psicopatologia Clínica pelo Laboratoire de Psychopathologie Clinique et Psychanalyse da Aix-Marseille Université (França); Livre-Docente em Psicopatologia do Departamento de Psicologia Médica e Psiquiatria da Faculdade de Ciências Médicas da Universidade Estadual de Campinas - Unicamp (Campinas, SP, Br), onde dirige o Laboratório de Psicopatologia: Sujeito e Singularidade (LaPSuS); Diretor do Núcleo de São Paulo do Corpo Freudiano - Escola de Psicanálise. Rua Tessália Vieira de Camargo, 126 - Cidade Universitária “Zeferino Vaz” 13083-887 Campinas, SP, Br. marioecpereira@uol.com.br https://orcid.org/0000-0002-7975-8863

This is an open-access article, which permits unrestricted use, distribution, and reproduction in any medium for non-commercial purposes provided the original authors and sources are credited. 